\title{
Mineral and volatile composition of água-mel from Portugal
}

\author{
Maria Graça Miguel $^{1} \cdot$ Smail Aazza $^{1}$ - Maria Dulce Antunes ${ }^{1} \cdot$ \\ Maria Leonor Faleiro ${ }^{2} \cdot$ José G. Barroso $^{3} \cdot$ Luis G. Pedro $^{3} \cdot$ A. Cristina Figueiredo ${ }^{3}$
}

Received: 1 June 2015 / Revised: 27 July 2015 / Accepted: 5 August 2015 / Published online: 25 August 2015

(C) Springer-Verlag Berlin Heidelberg 2015

\begin{abstract}
Água-mel (honey-water) is a typical honey-based product produced by the Portuguese beekeepers, particularly in southern Portugal. Água-mel was characterized by mineral content and volatiles contents. Mineral content evaluation was performed based on a random sampling of 14 samples from a total of 16 samples provided by local producers. Mineral content showed that potassium predominated in águamel samples (1270-4105 mg/kg). The concentration of aluminium in one sample was tenfold higher $(5.8 \mathrm{mg} / \mathrm{kg}$ ) than in the remaining samples $(0.3-0.6 \mathrm{mg} / \mathrm{kg})$. Água-mel volatiles were isolated by hydrodistillation and analysed by gas
\end{abstract}

Maria Graça Miguel

mgmiguel@ualg.pt

Smail Aazza

aazzasmail@ymail.com

Maria Dulce Antunes

mantunes@ualg.pt

Maria Leonor Faleiro

mfaleiro@ualg.pt

José G. Barroso

jmbarroso@fc.ul.pt

Luis G. Pedro

lmpedro@fc.ul.pt

A. Cristina Figueiredo

acsf@fc.ul.pt

1 Departamento de Química e Farmácia, Faculdade de Ciências e Tecnologia, Universidade do Algarve, MeditBio, Campus de Gambelas, 8005-139 Faro, Portugal

2 Faculdade de Ciências e Tecnologia, CBMR, Edf. 8, Campus de Gambelas, 8005-139 Faro, Portugal

3 Centro de Estudos do Ambiente e do Mar Lisboa, Faculdade de Ciências, 1749016 Lisboa, Portugal., Universidade de Lisboa, CBV, DBV, 1749-016 Lisbon, Portugal chromatography (GC) and gas chromatography-mass spectrometry (GC-MS) from a subset of eight samples. Cluster analysis showed two poorly correlated clusters $\left(S_{\text {corr }}<0.3\right)$. Cluster I only sample was dominated by trans- $\beta$-ocimene $(19 \%), \gamma$-terpinene $(15 \%)$ and 2-furfural $(9 \%)$. Cluster II that included the remaining seven samples showed two moderately correlated subclusters $\left(S_{\text {corr }}<0.5\right)$. The six samples with high correlation from subcluster IIa were dominated by 2-furfural (18-41\%) and benzene acetaldehyde (12-39\%). $n$-Nonadecane (14\%), $n$-heneicosane and 2-furfural (both $13 \%)$ were the main components of subcluster IIb sample. Although the presence of some volatile compounds can help in the correlation between água-mel and honey botanical source, the final product varies largely according to the preparation process even for the same producer, in different years. Água-mel detailed characterization may assist in bringing added value to this typical Portuguese honey-based product.

Keywords Portugal $\cdot$ Água-mel $\cdot$ Honey $\cdot$ Mineral composition $\cdot$ Volatiles

Abbreviations
GC $\quad$ Gas chromatography
GC-MS $\quad$ Gas chromatography-mass spectrometry

\section{Introduction}

For their importance in local economies, regional honeys and honey-based products are gaining increased interest [1-4]. The Portuguese beekeepers, particularly in southern Portugal (Algarve and Alentejo regions), produce for long a typical honey-based product called água-mel. The production of água-mel starts after the extraction of honey from the honeycombs. These honeycombs are then crumbled and dipped 
into warm water $\left(70{ }^{\circ} \mathrm{C}\right)$, and the remaining liquid constituted by the washing water, as well as honey, propolis and pollen residues is, afterwards, cooked during 9-12 h, until it forms a dark-golden brown liquid syrup, with $70^{\circ}-77^{\circ}$ Brix. In order to know that the right consistency is attained, a drop of the syrup should be dropped over a nail, making a small round ball $[5,6]$. This preparation procedure resembles the production of the Italian honey-based product abbamele $[1,2]$.

Água-mel is used in Portugal not only for folk medicinal purposes, to improve upper respiratory tract problems, but also in local gastronomy to spread in bread, for salads or fresh cheese dressing, as sweetener, and in the preparation of the typical bolo de água-mel (honey-water cake) [5, 6].

The physico-chemical characterization and microbiological quality of Portuguese água-mel as well as their antimicrobial, antiviral and antioxidant attributes were recently reported $[6,7]$. Changes in some physical and chemical parameters during the traditional process of production were also evaluated by Figueira and Cavaco [5].

The present study aimed at the valorization of this honey-based product by investigating água-mel volatiles and mineral composition and understanding the influence of the preparation procedure and honey type used on the final product characteristics.

\section{Materials and methods}

\section{Materials}

A total of 16 água-mel samples were kindly provided by local producers, as detailed in Table 1. For the volatiles

Table 1 Água-mel producer, year of production and code used in the volatiles analysis

\begin{tabular}{lll}
\hline Água-mel producer & Year of production & Code \\
\hline 1B & 2008 & 1B_2008 \\
1B & 2010 & 1B_2010 \\
1A & 2011 & 1A_2011 \\
1B & 2011 & 1B_2011 \\
1C & 2011 & 1C_2011 \\
1D & 2011 & 1D_2011 \\
1E & 2011 & 1E_2011 \\
1F & 2011 & 1F_2011 \\
1H & 2011 & 1H_2011 \\
1I & 2011 & 1I_2011 \\
1J & 2011 & 1J_2011 \\
1K & 2011 & 1K_2011 \\
1L & 2011 & 1L_2011 \\
1M & 2011 & 1M_2011 \\
1N & 2011 & 1N_2011 \\
\hline
\end{tabular}

analysis, a subset of eight samples was randomly selected from the total sampling (Table 1). Água-mel samples were kept at room temperature until analysis.

\section{Mineral content}

Água-mel samples $(5 \mathrm{~g})$ were submitted to calcination $\left(550{ }^{\circ} \mathrm{C}\right)$. After cooling, the residue was placed in a desiccator up to further analysis. Nitric acid $(5 \mathrm{~mL})$ was added to the água-mel ash, and the mixture was stirred on a heating plate to almost complete dryness. Thereafter, nitric acid (10 $\mathrm{mL})$ was added, and the mixture was brought up to $25 \mathrm{~mL}$ with distilled water. From these solutions, the mineral content ( $\mathrm{Na}, \mathrm{K}, \mathrm{Mg}, \mathrm{Mn}, \mathrm{Zn}$ and $\mathrm{Fe}$ ) was determined by flame atomic absorption spectrometry (air-acetylene) using a PerkinElmer Aanalyst 800, except aluminium and cupper which were analysed by graphite furnace atomic absorption spectrometry. Results were expressed as milligrams of mineral content per kilograms of água-mel.

\section{Volatiles extraction}

Água-mel volatiles were isolated by hydrodistillation for $3 \mathrm{~h}$ using a Clevenger-type apparatus according to the European Pharmacopoeia method [8]. The isolation procedure was run at a distillation rate of $3 \mathrm{ml} / \mathrm{min}$. The extracted volatiles were recovered in distilled $n$-pentane, collected in a vial and concentrated to a minimum volume, at room temperature, under nitrogen flux. The volatiles were stored at $-20{ }^{\circ} \mathrm{C}$ in the dark until analysis.

\section{Gas chromatography (GC)}

Gas chromatographic analyses were performed using a PerkinElmer Clarus 400 gas chromatograph equipped with two flame ionization detectors, a data handling system and a vaporizing injector port into which two columns of different polarities were installed: a DB-1 fused-silica column (polydimethylsiloxane, $30 \mathrm{~m} \times 0.25 \mathrm{~mm}$ i.d., film thickness $0.25 \mu \mathrm{m}$; J \& W Scientific Inc., Rancho Cordova, CA, USA) and a DB-17HT fused-silica column [(50\% phenyl)-methylpolysiloxane, $30 \mathrm{~m} \times 0.25 \mathrm{~mm}$ i.d., film thickness $0.15 \mu \mathrm{m}$; J \& W Scientific Inc.]. Oven temperature was programmed, $45-175{ }^{\circ} \mathrm{C}$, at $3{ }^{\circ} \mathrm{C} \mathrm{min}{ }^{-1}$, subsequently at $15{ }^{\circ} \mathrm{C} \min ^{-1}$ up to $300{ }^{\circ} \mathrm{C}$, and then held isothermal for $10 \mathrm{~min}$; injector and detector temperatures, 280 and $300{ }^{\circ} \mathrm{C}$, respectively; carrier gas, hydrogen, adjusted to a linear velocity of $30 \mathrm{~cm} \mathrm{~s}^{-1}$. The samples were injected using split sampling technique, ratio 1:50. The volume of injection was $0.1 \mu \mathrm{L}$ of a pentane-volatiles solution $(1: 1)$. The percentage composition of the volatiles was computed by the normalization method from the GC peak areas, calculated as mean values of two injections from each sample, 
using the response factors reported in the literature as previously detailed [9].

\section{Gas chromatography-mass spectrometry (GC-MS)}

The GC-MS unit consisted of a PerkinElmer Clarus 600 gas chromatograph, equipped with DB-1 fused-silica column $(30 \mathrm{~m} \times 0.25 \mathrm{~mm}$ i.d., film thickness $0.25 \mu \mathrm{m}$; J \& W Scientific, Inc.) and interfaced with a PerkinElmer 600T mass spectrometer (software version 5.4, PerkinElmer, Shelton, CT, USA). Injector and oven temperatures were as above; transfer line temperature, $280{ }^{\circ} \mathrm{C}$; ion source temperature, $220{ }^{\circ} \mathrm{C}$; carrier gas, helium, adjusted to a linear velocity of $30 \mathrm{~cm} \mathrm{~s}^{-1}$; split ratio, 1:40; ionization energy, $70 \mathrm{eV}$; scan range, 40-300 u; scan time, $1 \mathrm{~s}$. The identity of the components was assigned by comparison of their retention indices, relative to $\mathrm{C}_{8}-\mathrm{C}_{28} n$-alkane indices and GC-MS spectra from a home-made library, created with reference essential oils (REO), laboratory-synthesized components (LSC), laboratory-isolated compounds (LIC) and commercially available standards (CAS).

\section{Statistical analysis}

The percentage composition of the isolated volatiles was used to determine the relationship between the samples by cluster analysis using Numerical Taxonomy Multivariate Analysis System (NTSYS-pc software, version 2.2, Exeter Software, Setauket, New York) [10]. For cluster analysis, correlation coefficient was selected as a measure of similarity among all accessions, and the Unweighted Pair Group Method with Arithmetical Averages (UPGMA) was used for cluster definition. The degree of correlation was evaluated, according to Pestana and Gageiro [11], as very high
(0.9-1), high (0.7-0.89), moderate $(0.4-0.69)$, low $(0.2-$ $0.39)$ and very low $(<0.2)$.

\section{Results and discussion}

\section{Mineral content}

Potassium (K) predominated $(1270-4105 \mathrm{mg} / \mathrm{kg})$ in the mineral content of all água-mel samples (Table 2), although with great variability depending on the producer. The predominance of potassium was expected since this element is quantitatively the most important mineral in honey [12-14]. However, some values were much higher than those reported for honey samples, such as for 1N_2011 $(3110 \mathrm{mg} / \mathrm{kg})$ and 1F_2011 (4105 mg/kg). Sodium was the second more important element, whose concentrations ranged from 109.5 to $416.5 \mathrm{mg} / \mathrm{kg}$. Aluminium (Al) was tenfold higher in the sample 1N_2011 when compared to the remaining samples (Table 2). Iron (Fe) content $(61.5 \mathrm{mg} / \mathrm{kg})$ was also higher in the same sample than in the remaining ones $(15.0-55.5 \mathrm{mg} / \mathrm{kg}$ ) (Table 2). The levels of copper $(\mathrm{Cu})$ were also different between samples, from a minimal concentration around $4 \mathrm{mg} / \mathrm{kg}$ in several samples to $10.5-10.6 \mathrm{mg} / \mathrm{kg}$ in 1E_2011 and 1O_2011, respectively. Other example of great variability was magnesium $(\mathrm{Mg})$ whose contents ranged from $37.9 \mathrm{mg} / \mathrm{kg}$, in 1K_2011 sample, to $188.9 \mathrm{mg} / \mathrm{kg}$, in 1I_2011 (Table 2).

Variations in trace element contents in different honey types have been correlated with its botanical origin [15, 16]. Although água-mel mineral content variability found in the present work can also be linked to this factor, it is important to stress that the highest content of aluminium or iron in one sample may also be attributed to the traditional

Table 2 Mineral contents $(\mathrm{mg} / \mathrm{kg}) \pm$ standard deviation of Portuguese Água-mel

\begin{tabular}{|c|c|c|c|c|c|c|c|c|}
\hline Água-mel samples & $\mathrm{Al}$ & $\mathrm{Cu}$ & $\mathrm{Fe}$ & $\mathrm{K}$ & $\mathrm{Mg}$ & Mn & $\mathrm{Zn}$ & $\mathrm{Na}^{\mathrm{a}}$ \\
\hline 1A_2011 & $0.4 \pm 0.2$ & $6.5 \pm 0.3$ & $30.5 \pm 1.0$ & $2600 \pm 460$ & $101.1 \pm 0.7$ & $4.8 \pm 1.5$ & $4.2 \pm 0.3$ & 121.5 \\
\hline 1B_2011 & $0.5 \pm 0.1$ & $7.8 \pm 0.5$ & $36.5 \pm 1.5$ & $2900 \pm 25$ & $78.8 \pm 0.2$ & $5.6 \pm 1.8$ & $3.9 \pm 0.7$ & 117.0 \\
\hline 1C_2011 & $0.3 \pm 0.2$ & $5.4 \pm 0.4$ & $26.0 \pm 1.0$ & $2350 \pm 165$ & $93.6 \pm 0.8$ & $3.9 \pm 0.5$ & $3.1 \pm 0.2$ & 253.5 \\
\hline 1D_2011 & $0.5 \pm 0.3$ & $9.1 \pm 0.7$ & $15.0 \pm 2.5$ & $1625 \pm 65$ & $45.8 \pm 0.2$ & $4.9 \pm 0.8$ & $3.8 \pm 0.1$ & 109.5 \\
\hline 1E_2011 & $0.5 \pm 0.1$ & $10.5 \pm 0.3$ & $25.0 \pm 1.0$ & $2310 \pm 40$ & $60.5 \pm 0.1$ & $5.8 \pm 0.5$ & $4.9 \pm 0.4$ & 203.0 \\
\hline 1F_2011 & $0.4 \pm 0.2$ & $5.2 \pm 0.6$ & $19.0 \pm 2.5$ & $4105 \pm 45$ & $55.3 \pm 0.8$ & $2.8 \pm 1.0$ & $3.0 \pm 0.1$ & 213.0 \\
\hline 1H_2011 & $0.5 \pm 0.1$ & $9.0 \pm 1.2$ & $39.5 \pm 0.5$ & $1620 \pm 215$ & $163.1 \pm 0.3$ & $3.7 \pm 0.3$ & $3.5 \pm 0.2$ & 241.5 \\
\hline 1I_2011 & $0.6 \pm 0.2$ & $4.4 \pm 0.4$ & $20.0 \pm 1.5$ & $1735 \pm 110$ & $188.9 \pm 0.4$ & $5.3 \pm 0.7$ & $3.6 \pm 0.2$ & 141.0 \\
\hline 1J_2011 & $0.6 \pm 0.2$ & $5.6 \pm 0.7$ & $29.0 \pm 0.5$ & $1270 \pm 40$ & $145.6 \pm 0.1$ & $5.1 \pm 0.4$ & $4.1 \pm 0.1$ & 143.0 \\
\hline 1K_2011 & $0.4 \pm 0.1$ & $4.3 \pm 0.2$ & $55.5 \pm 3.0$ & $1485 \pm 45$ & $37.9 \pm 0.7$ & $3.9 \pm 0.4$ & $3.2 \pm 0.2$ & 181.5 \\
\hline 1L_2011 & $0.4 \pm 0.1$ & $4.9 \pm 0.4$ & $26.5 \pm 0.5$ & $2055 \pm 5$ & $44.5 \pm 0.2$ & $4.2 \pm 0.5$ & $3.8 \pm 0.1$ & 416.5 \\
\hline 1M_2011 & $0.4 \pm 0.1$ & $6.0 \pm 0.2$ & $30.0 \pm 1.0$ & $1625 \pm 250$ & $96.3 \pm 1.8$ & $3.0 \pm 0.9$ & $4.1 \pm 0.1$ & 191.0 \\
\hline 1N_2011 & $5.8 \pm 0.9$ & $5.5 \pm 0.1$ & $61.5 \pm 1.0$ & $3110 \pm 60$ & $77.7 \pm 0.3$ & $5.5 \pm 0.8$ & $2.8 \pm 0.25$ & 215.0 \\
\hline
\end{tabular}

a Due to sample shortage, only one measurement was made for $\mathrm{Na}$ 
Table 3 Percentage composition of the components identified in the volatiles isolated by hydrodistillation from eight samples of Água-mel obtained from local producers (Table 1)

\begin{tabular}{|c|c|c|c|c|c|c|c|c|c|c|c|c|c|}
\hline \multirow[t]{4}{*}{ Components } & \multirow[t]{4}{*}{ RI } & \multirow[t]{4}{*}{ IP } & \multirow[t]{4}{*}{$\mathrm{REO}^{\mathrm{a}}$} & \multirow[t]{4}{*}{$\mathrm{REO}^{\mathrm{b}}$} & \multirow[t]{4}{*}{$\mathrm{RI}^{\mathrm{c}, \mathrm{d}}$} & \multirow{4}{*}{$\begin{array}{l}\text { Cluster } \\
\text { I } \\
1 B_{-} \\
2008\end{array}$} & \multicolumn{7}{|c|}{ Cluster II } \\
\hline & & & & & & & \multicolumn{6}{|l|}{ IIa } & \multirow{2}{*}{$\begin{array}{l}\mathrm{IIb} \\
1 \mathrm{E}_{-}\end{array}$} \\
\hline & & & & & & & $1 \mathrm{~B}_{-}$ & $1 \mathrm{~K}_{-}$ & $1 \mathrm{~B}_{-}$ & $1 \mathrm{~F}_{-}$ & $1 \mathrm{H}_{-}$ & $1 C_{-}$ & \\
\hline & & & & & & & 2010 & 2011 & 2011 & 2011 & 2011 & 2011 & 2011 \\
\hline$n$-Octane & 800 & CAS 2 & & & & 1.2 & 1.4 & 2.1 & 0.9 & 0.3 & 2.4 & 3.9 & 4.5 \\
\hline 2-Furfural & 825 & CAS 2 & & & 829 & 9.1 & 35.9 & 34.2 & 12.7 & 41.4 & 36.1 & 38.6 & 17.8 \\
\hline Protoanemonine & 854 & & & & & & & & & 2 & $t$ & & \\
\hline$p$-Xylene & 855 & CAS 2 & & & 857 & & & & & & & 2.9 & 2.8 \\
\hline$o$-Xylene & 856 & CAS 2 & & & 869 & & & & & & & 5.7 & 0.2 \\
\hline 2-Acetyl furan ${ }^{\mathrm{e}}$ & 900 & & & & 892 & 1 & 5 & 1.5 & 1.1 & 5 & 6 & 0.2 & 1.8 \\
\hline$n$-Nonane & 900 & CAS 2 & & & & & & & & & & 2.5 & \\
\hline$\alpha$-Thujene & 924 & CAS 1 & 922 & 922 & 929 & 1.5 & & & & & & & \\
\hline Benzaldehyde & 927 & CAS 2 & & & 934 & & 0.8 & 0.1 & 0.5 & & 1 & 1.1 & 0.5 \\
\hline$\alpha$-Pinene & 930 & CAS 1 & 930 & 930 & 931 & 0.4 & & & & & & & \\
\hline 5-Methyl furfural & 938 & & & & 934 & 0.3 & 0.7 & 0.5 & 3.2 & 3.7 & 5.3 & 4.5 & 0.5 \\
\hline Camphene & 938 & CAS 1 & 938 & 942 & 938 & $t$ & & & & & & & \\
\hline Sabinene & 958 & CAS 1 & 958 & 964 & 959 & 0.6 & 1.7 & 1.5 & & & & & \\
\hline 1-Octen-3-ol & 961 & CAS 2 & 961 & 959 & 972 & $t$ & & & & & & & \\
\hline$\beta$-Pinene & 963 & CAS 1 & 963 & 970 & 962 & 2.3 & 8.9 & & & & & & \\
\hline Hexanoic acid (= Caproic acid) & 968 & CAS 2 & & & 981 & $t$ & & & & & & & \\
\hline$\beta$-Myrcene & 975 & CAS 2 & 975 & 980 & 981 & 2.3 & $t$ & 0.3 & $t$ & & & & \\
\hline$\alpha$-Phellandrene & 995 & CAS 2 & 1000 & 998 & 986 & 0.2 & & 0.1 & & & & & \\
\hline Benzyl alcohol & 1000 & CAS 2 & & & 1004 & $t$ & $t$ & $t$ & & & & & \\
\hline Benzene acetaldehyde & 1002 & CAS 2 & & & 1006 & 2.3 & 12.2 & 14.2 & 2.8 & 16.7 & 29.7 & 24.1 & 39.2 \\
\hline 2,6,6-Trimethyl cyclohexanone & 1003 & CAS 2 & & & 1016 & & & & & & $t$ & & $t$ \\
\hline$p$-Cymene & 1003 & CAS 2 & 1003 & 1011 & 1004 & 2.4 & 1.9 & $t$ & $t$ & & & $t$ & \\
\hline 1,8-Cineole & 1005 & CAS 2 & 1005 & & 1010 & 5.5 & & $t$ & & 0.2 & & & \\
\hline Limonene & 1009 & CAS 3 & 1009 & 1020 & 1014 & 1.6 & 0.3 & 5 & 4.1 & 0.1 & & & \\
\hline cis- $\beta$-Ocimene & 1017 & CAS 3 & 1017 & 1025 & 1015 & 4.6 & & 0.1 & & & & & \\
\hline Acetophenone & 1017 & CAS 2 & & & 1036 & $t$ & & $t$ & & $t$ & & & \\
\hline trans- $\beta$-Ocimene & 1027 & CAS 3 & 1027 & 1035 & 1026 & 19 & & 0.1 & & & & & \\
\hline$\gamma$-Terpinene & 1035 & CAS 2 & 1035 & 1046 & 1049 & 14.6 & 0.4 & 0.1 & 0.7 & & & $t$ & 0.1 \\
\hline trans-Sabinene hydrate & 1037 & & 1037 & & 1052 & $t$ & & & & & & & \\
\hline cis-Linalool oxide & 1045 & & & & 1078 & $t$ & 0.1 & 0.2 & & 0.5 & & 3.2 & 0.1 \\
\hline 2-Methyl decane ${ }^{\mathrm{e}}$ & 1046 & & & & 1065 & $t$ & 0.1 & 0.1 & $t$ & 0.1 & 0.8 & 0.1 & 0.1 \\
\hline Fenchone & 1050 & CAS 2 & & & 1065 & 0.5 & & & & & & & \\
\hline trans-Linalool oxide & 1059 & & & & 1064 & & & 0.1 & & $t$ & & 1.5 & \\
\hline Terpinolene & 1064 & CAS 2 & 1064 & 1077 & 1077 & $t$ & & 1.1 & & & & & \\
\hline Phenyl ethyl alcohol & 1064 & CAS 2 & & & 1074 & 0.4 & $t$ & 0.1 & & & & $t$ & 0.1 \\
\hline Linalool & 1074 & CAS 2 & 1074 & 1082 & 1082 & 0.5 & & 0.1 & & & & & \\
\hline Isophorone & 1074 & CAS 2 & & & 1074 & & & 0.1 & & & & & \\
\hline$n$-Undecane & 1100 & CAS 2 & & & & $t$ & & 0.3 & $t$ & $t$ & 0.1 & $t$ & 0.1 \\
\hline 2-Ethyl hexanoic acid ${ }^{\mathrm{e}}$ & 1101 & & & & 1097 & 2.5 & & 0.2 & & & & & \\
\hline Borneol & 1134 & CAS 2 & 1134 & & 1140 & $t$ & & & & & & & \\
\hline Menthol & 1148 & CAS 2 & & & 1150 & $t$ & & & & & & & \\
\hline Terpinen-4-ol & 1148 & CAS 2 & 1148 & 1158 & 1171 & 0.5 & $t$ & 0.1 & & & & & \\
\hline$p$-Cymen-8-ol & 1148 & & 1148 & 1158 & 1171 & & & & & $t$ & & & \\
\hline Octanoic acid & 1152 & CAS 2 & & & 1173 & $t$ & & & & & & & \\
\hline
\end{tabular}


Table 3 continued

\begin{tabular}{|c|c|c|c|c|c|c|c|c|c|c|c|c|c|}
\hline \multirow[t]{4}{*}{ Components } & \multirow[t]{4}{*}{ RI } & \multirow[t]{4}{*}{ IP } & \multirow[t]{4}{*}{$\mathrm{REO}^{\mathrm{a}}$} & \multirow[t]{4}{*}{$\mathrm{REO}^{\mathrm{b}}$} & \multirow[t]{4}{*}{$\mathrm{RI}^{\mathrm{c}, \mathrm{d}}$} & \multirow{4}{*}{$\begin{array}{l}\text { Cluster } \\
\text { I } \\
1 B_{-} \\
2008\end{array}$} & \multicolumn{7}{|c|}{ Cluster II } \\
\hline & & & & & & & \multicolumn{6}{|l|}{ IIa } & \multirow{2}{*}{$\begin{array}{l}\mathrm{IIb} \\
1 \mathrm{E}_{-}\end{array}$} \\
\hline & & & & & & & $1 \mathrm{~B}_{-}$ & $1 \mathrm{~K}_{-}$ & $1 \mathrm{~B}_{-}$ & $1 \mathrm{~F}_{-}$ & $1 \mathrm{H}_{-}$ & $1 C_{-}$ & \\
\hline & & & & & & & 2010 & 2011 & 2011 & 2011 & 2011 & 2011 & 2011 \\
\hline$\alpha$-Terpineol & 1159 & CAS 3 & 1159 & 1169 & 1157 & 0.6 & 2.1 & 0.1 & 0.7 & & & & \\
\hline Methyl chavicol & 1163 & & & & 1180 & $t$ & & & & & & & \\
\hline cis-Cinnamaldehyde & 1169 & CAS 2 & & & 1184 & & & & & 0.2 & & & \\
\hline Cumin aldehyde & 1200 & CAS 2 & & & 1215 & 0.1 & $t$ & & & & & & \\
\hline$p$-Anisaldehyde $\mathrm{e}^{\mathrm{e}}$ & 1200 & & & & 1213 & 0.2 & 0.5 & $t$ & $t$ & & & & $t$ \\
\hline Thymol methyl ether & 1210 & CAS 2 & & & 1207 & 0.1 & & & & & & & \\
\hline Butyrophenone & 1212 & CAS 2 & & & & 0.2 & $t$ & & & & & & \\
\hline Carvacrol methyl ether & 1224 & CAS 2 & & & 1223 & 2.1 & & & & & & & \\
\hline trans-Cinnamaldehyde & 1224 & CAS 2 & & & 1239 & $t$ & & 0.1 & $t$ & 8.1 & & & \\
\hline Linalyl acetate & 1245 & CAS 2 & & & 1239 & $t$ & & & & & & & \\
\hline Trimethyl phenol ${ }^{\mathrm{e}}$ (isomer not identified) & 1247 & & & & & 0.7 & 3.1 & 6.7 & 4.2 & 0.1 & 1.2 & 1.4 & 3.5 \\
\hline Anisyl formate $e^{e}$ & 1251 & & & & 1300 & 0.4 & 9.1 & 14.8 & 0.5 & 0.1 & 0.1 & 0.1 & $t$ \\
\hline trans-Anethole & 1254 & CAS 2 & & & 1270 & 0.3 & & & & & & & \\
\hline trans-Cinnamyl alcohol & 1268 & CAS 2 & & & 1268 & & & 0.2 & & & & & \\
\hline Thymol & 1275 & & 1275 & & 1290 & 5.6 & 0.4 & 0.2 & & 0.5 & 0.5 & 0.3 & 2 \\
\hline Carvacrol & 1286 & CAS 2 & 1286 & & 1298 & 3.7 & & 0.3 & & 0.1 & 0.3 & 0.1 & $t$ \\
\hline Trimethyl phenol ${ }^{\mathrm{e}}$ (isomer not identified) & 1295 & & & & & 0.4 & 2.9 & 8.1 & 2 & 0.6 & 0.2 & 0.6 & $t$ \\
\hline Methyl eugenol & 1377 & CAS 2 & & & 1374 & $t$ & & 0.1 & & & & & \\
\hline Coumarin & 1391 & & & & 1397 & & & & & 3 & & & \\
\hline$\beta$-Caryophyllene & 1414 & CAS 2 & 1414 & 1408 & $1415 / 1421$ & 3.9 & & 0.1 & & & & & \\
\hline$\alpha$-Humulene & 1447 & CAS 3 & 1447 & 1442 & $1439 / 1455$ & 0.5 & & & & & & & \\
\hline Germacrene D & 1474 & & 1474 & 1467 & $1474 / 1479$ & 1 & & $t$ & & & & & \\
\hline Bicyclogermacrene & 1487 & & 1487 & & $1490 / 1494$ & 0.8 & & 0.4 & & & & & \\
\hline trans,trans- $\alpha$-Farnesene & 1495 & CAS 2 & & & $1509 / 1498$ & 0.5 & & & & & & & \\
\hline$n$-Pentadecane & 1500 & CAS 2 & & & & & & 0.1 & & & & & \\
\hline$\beta$-Bisabolene & 1500 & & & & $1511 / 1503$ & 0.6 & & & & & & & \\
\hline$\delta$-Cadinene & 1505 & & 1505 & 1508 & $1513 / 1520$ & $t$ & & & & & & & \\
\hline Spathulenol & 1551 & & & & 1552 & $t$ & & & & & & & \\
\hline$\beta$-Caryophyllene oxide & 1561 & CAS 3 & 1561 & 1557 & 1565 & $t$ & & & & & & & \\
\hline Viridiflorol & 1569 & & 1569 & & 1568 & $t$ & & & & & & & \\
\hline$\gamma$-Eudesmol & 1609 & & & & 1626 & & & 0.2 & & & & & \\
\hline cis-Methyl dihydrojasmonate & 1616 & & & & 1656 & $t$ & & 0.1 & & & & & \\
\hline epi- $\alpha$-Muurolol & 1616 & & 1616 & 1616 & 1616 & $t$ & & 0.1 & & & & & \\
\hline$\alpha$-Cadinol & 1626 & & 1626 & 1627 & 1637 & $t$ & & & & & 0.3 & & \\
\hline$n$-Pentadecanal & 1688 & & & & 1687 & $t$ & & & & & & & \\
\hline$n$-Heptadecane & 1700 & CAS 2 & & & & & & & $t$ & & & & \\
\hline Benzyl benzoate & 1701 & CAS 2 & & & 1723 & $t$ & 0.6 & 0.2 & & & & & \\
\hline$n$-Nonadecane & 1900 & CAS 2 & & & & & & & 14.2 & & & & \\
\hline Hexadecanoic acid (= Palmitic acid) & 1908 & CAS 2 & & & 1942 & 0.7 & 1.2 & 0.3 & $t$ & 0.6 & 0.6 & 0.3 & 5.2 \\
\hline$n$-Octadecanol (= Stearyl alcohol) & 2071 & & & & 2067 & & & & 3.2 & & 0.1 & & 2.5 \\
\hline$n$-Heneicosane & 2100 & CAS 2 & & & & 0.5 & 1 & 0.1 & 12.9 & & 0.7 & 0.3 & 11.6 \\
\hline Linoleic acid ethyl ester (= ethyl linoleate) & 2137 & LSC & & & 2140 & & & & 0.6 & 0.9 & & & \\
\hline$n$-Docosane & 2200 & CAS 2 & & & & & & & 0.5 & & & & \\
\hline$n$-Eicosanol & 2265 & & & & 2267 & & & & 0.8 & & & & \\
\hline$n$-Tricosane & 2300 & CAS 2 & & & & & & 0.1 & 6.4 & & & & \\
\hline
\end{tabular}


Table 3 continued

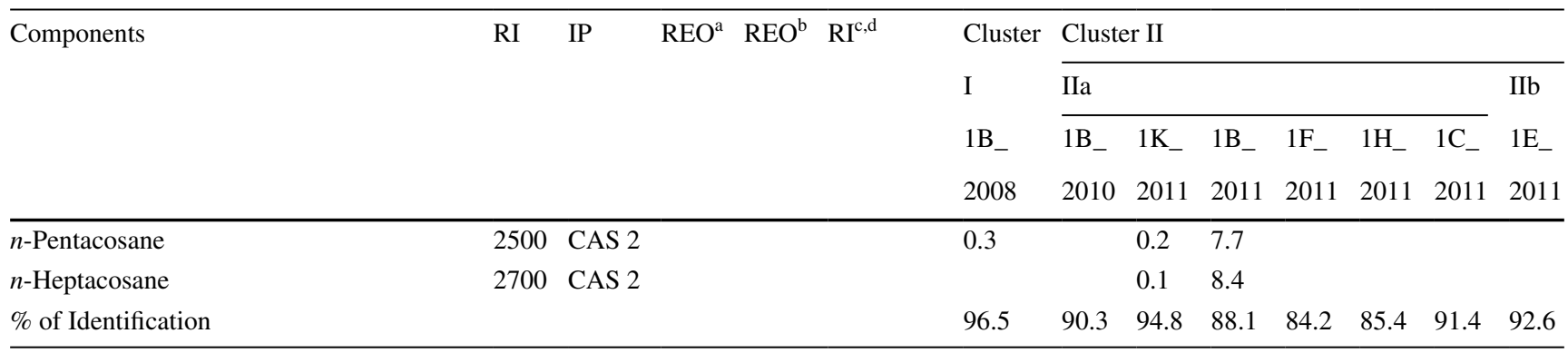

For samples grouped on each of the clusters I-II and subclusters a-b, see Fig. 1

$\mathrm{RI}=$ retention index calculated relative to $\mathrm{C}_{9}-\mathrm{C}_{27} n$-alkanes on the DB- 1 column; $\mathrm{IP}=$ identification procedure. All components were identified based on a laboratory-made library created with reference essential oils (REO), laboratory-synthesized components (LSC), laboratory-isolated compounds (LIC) and commercially available standards (CAS)

a,b REO, reference essential oils of Thymus caespititius [21] and Juniperus cedrus [22], in which components' identity was confirmed by RI, GC-MS and ${ }^{13}$ C-NMR. CAS 1. Extrasynthese (Cymit Química, S.L.), CAS 2. Sigma-Aldrich, CAS 3. Fluka, CAS 4. Riedel-de Haën. Unless otherwise specified, retention index from LSC, LIC and CAS is that reported in previous column. ${ }^{c, d}$ RI, regular font values from Linstrom and Mallard [23], italic values from Joulain and Köning [24]. ${ }^{c}$ RI, literature retention indices on DB-1 or similar phase column (100\% dimethylpolysiloxane) not from the authors' laboratory. ${ }^{\mathrm{d}} \mathrm{RI}$, literature retention indices on a Cp-Sil 5 (100\% dimethylpolysiloxane). t, trace $(<0.05 \%)$.

e Tentative identification based on mass spectra only

way to produce água-mel, namely to the type of containers where água-mel was produced and/or stored.

\section{Volatiles evaluation}

The volatile fraction isolated from each individual of águamel sample was a complex mixture in which 91 components were identified, representing 84-97\% of the total volatiles. The identified volatile components are listed in Table 3 in the order of their elution on the DB- 1 column, arranged according to the total three types of volatile oils obtained by agglomerative cluster analysis.

Cluster analysis, based on água-mel volatiles composition, showed two poorly correlated clusters $\left(S_{\text {corr }}<0.3\right)$ (Fig. 1). Cluster I which included only one sample from 2008 was characterized by the dominance of trans- $\beta$ ocimene (19\%), $\gamma$-terpinene $(15 \%)$ and 2-furfural $(9 \%)$. Cluster II that included the remaining seven samples showed two moderately correlated subclusters $\left(S_{\text {corr }}<0.5\right)$. The six more correlated samples from subcluster IIa were dominated by 2 -furfural (18-41 \%) and benzene acetaldehyde (12-39\%). $n$-Nonadecane (14\%), $n$-heneicosane and 2 -furfural (both $13 \%$ ) were the main components of the one sample from subcluster IIb.

Benzene acetaldehyde, a common aromatic compound in honey samples [17-19], was present in all água-mel samples in a range of 2-39\% (Table 3). In honey, this variability has been related to the floral origin of honeys [17]. Generally, beekeepers use mixtures of honeys for água-mel production; thus, different proportions of several unifloral or even multifloral honeys may be responsible for that percentage range. 2-Furfural was also present in relative high amounts in all samples (9-41\%). This compound has also been reported as part of the volatile fraction of honey [20]. Other thermally derived furan derivatives were also present in relative abundance, such as 2-acetyl furan $(0.2-5 \%)$ and 5-methylfurfural (0.3-5\%) (Table 3).

$n$-Nonadecane, hexadecanoic acid, n-octadecanol, $n$-heneicosane, linoleic acid ethyl ester, $n$-docosane, $n$-eicosanol, $n$-tricosane, $n$-pentacosane and $n$-heptacosane present in relative high amounts in some água-mel samples may be due to the use of the honeycombs-derived beeswax. These compounds were also reported by Jerković et al. [2] in abbamele, from Sardinia (Italy), an água-mel similarly obtained product, by traditional honeycombs processing.

Despite the similarities of producing process, between abbamele [2] and the presently studied água-mel samples, there are some differences in the volatile profile. Limonene was the main monoterpene found in some samples of abbamele, which according to the authors could be attributed to the citrus rind addition during production process. In the present work, only one sample of água-mel had relatively high amounts of monoterpenes (1B_2008).

Among monoterpenes, trans- $\beta$-ocimene (19\%), $\gamma$-terpinene $(15 \%), 1,8$-cineole $(6 \%)$ and thymol $(6 \%)$ were the most relevant, present in percentages $\geq 5 \%$ in sample 1B_2008 (Table 3). However, in samples 1B_2010 and 1B_2011, 2 and 3 years later samples from the same beekeeper, these monoterpenes did not attain such high percentages (varying from not detected to $1 \%$, in the four samples). Instead, $\beta$-pinene was the major monoterpene in 1B_2010 sample (9 \%) and not detected in 1B_2011. Conversely, in 1B_2011 água-mel sample of the same producer, limonene (4\%) was the main monoterpene, whereas in 1B_2008 and 1B_2010, the percentages were 
Fig. 1 Dendrogram obtained by cluster analysis of the percentage composition of volatiles from Água-mel samples based on correlation and using UPGMA. For abbreviations, see Table 1

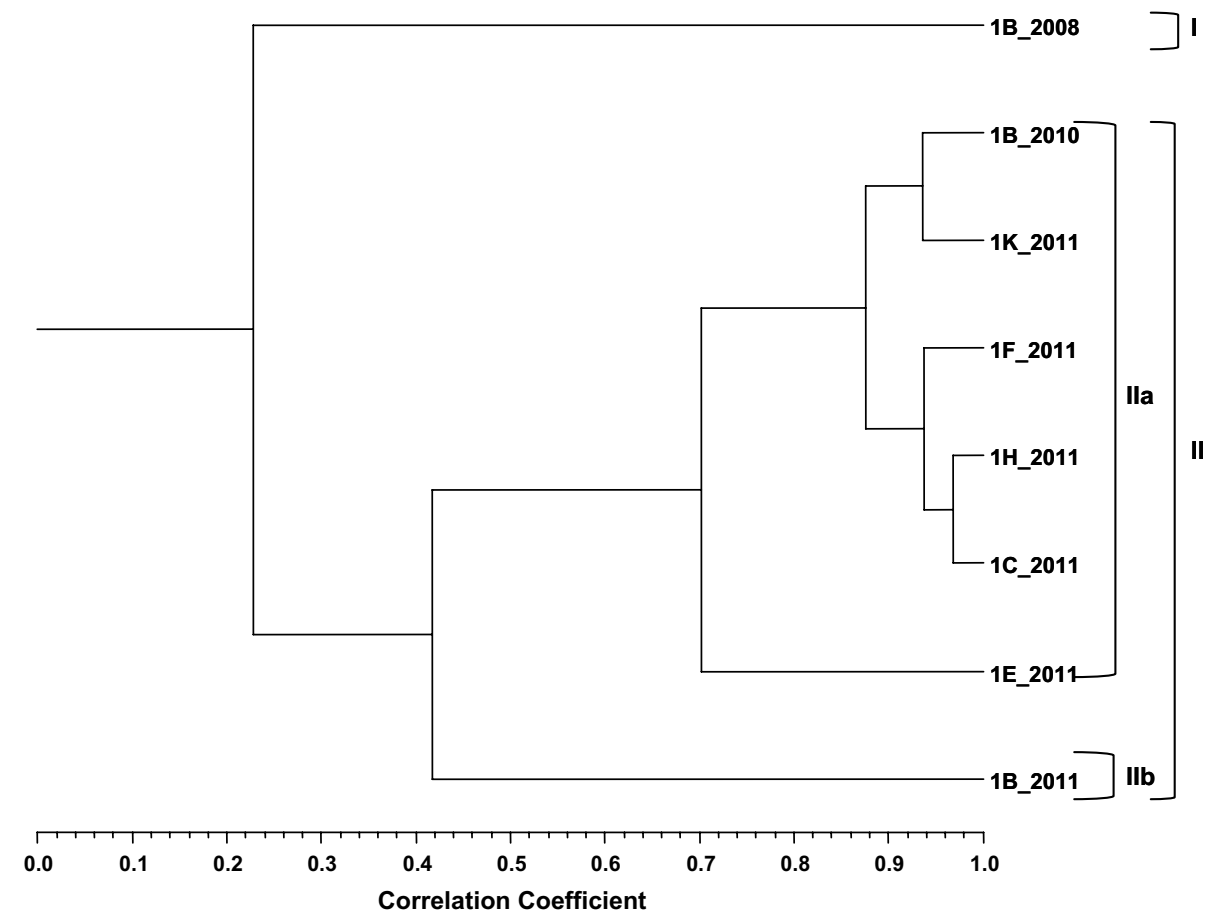

2 and $0.3 \%$, respectively. Limonene was also present as major monoterpene component $(5 \%)$ in sample $1 \mathrm{~K} \_2011$ (Table 3).

This variability was also found [6] for total phenols and flavonoids in the same samples. In that work, the authors concluded that these metabolites as well as antioxidant activity, measured through several methods were dependent on the producer and year of production. However, the authors also found that the antioxidant activity correlated better with the melanoidin content than with polyphenol content, particularly between melanoidin content and capacity for scavenging peroxyl radicals; between melanoidin content and capacity for scavenging nitric oxide radicals; and between melanoidin content and capacity for chelating metal ions [6]. Such results revealed that beyond the components constituting the honeys used for producing água-mel, there are other ones that are formed during its production which are important on the biological properties. In this way, the botanical origin of honeys along with the mode of production is particularly important on the final characteristics of água-mel.

Such as previously observed [6] for polyphenols, melanoidins and biological properties, in the present work it was possible to conclude that although the presence of some volatile compounds can help in the correlation between água-mel and honey botanical source, the final product also varies largely according to the preparation process (time of decoction, type of container, optional addition of spices, or other flavourings substances) even for the same producer, in different years.

\section{Conclusion}

This study reports the first mineral content and volatiles study of água-mel. Mineral content was similar to that generally found in honey. Potassium was the most abundant, nevertheless with significant differences between producers. Other elements such as $\mathrm{Al}, \mathrm{Cu}, \mathrm{Fe}$ and $\mathrm{Mg}$ also varied greatly. These differences may be attributed to the botanical origin of honeys used for água-mel production, but also to the type of containers used in its production.

Thermally derived furan derivatives were detected in água-mel samples, as a consequence of the prolonged decoction process, as well as some aromatic compounds frequent in honey and in beeswax. Their percentages, as well as other volatile compounds, like monoterpenes, varied according to the producer and, for the same producer, with production year.

The relative high concentrations of some minerals as well as those of the derived furan derivatives in água-mel need to be solved, and for this purpose, it will be necessary to standardize the production technology using stainless steel vessels and reducing the time of high-temperature heating.

Aiming at adding value to água-mel, gaining an in-depth knowledge on this a Portuguese typical honey-based product may support strategies for creation of new market trades. 
Acknowledgments This study was partially funded by Ministério da Agricultura, Mar, Ambiente e Ordenamento do Território (Portugal) under research contract Programa Apícola Nacional 2011-2013, Medida 6A, and by Fundação para a Ciência e a Tecnologia (FCT), under Pest-OE/EQB/LA0023/2011 and UID/AMB/50017/2013.

\section{Compliance with ethical standards}

\section{Conflict of interest None.}

Compliance with Ethics Requirements This article does not contain any studies with human or animal subjects.

\section{References}

1. Spano N, Ciulu M, Floris I, Panzanelli A, Pilo MI, Piu PC, Scanu R, Sanna G (2008) Food Chem 108(1):81-85

2. Jerković I, Kasum A, Marijanović Z, Tuberoso CIG (2011) Food Chem 124(1):401-410

3. Tornuka F, Karaman S, Ozturk I, Toker OS, Tastemur B, Sagdic O, Dogan M, Kayacier A (2013) Ind Crops Prod 46(1):124-131

4. Čanadanović-Brunet J, Ćetković G, Šaponjac VT, Stajčić S, Vulić J, Djilas S, Štajnerb D, Popović B (2014) Ind Crops Prod 62(1):1-7

5. Figueira AC, Cavaco T (2012) J Food Process Preserv 36(4):285-290

6. Miguel MG, Antunes MD, Aazza S, Duarte J, Faleiro ML (2013) Ital J Food Sci 25:275-282

7. Miguel MG, Faleiro L, Antunes MD, Aazza S, Duarte J, Silvério AR (2013) Food Chem Toxicol 56(1):136-144

8. Council of Europe (COE) European Directorate for the Quality of Medicines (2007) European Pharmacopoeia 6th edn. Strasbourg, France

9. Figueiredo AC, Garcia C, Sim-Sim M, Sérgio C, Pedro LG, Barroso JG (2010) Flavour Fragr J 25(4):219-222
10. Rohlf JF NTSYS-pc (2000) Numerical Taxonomy and Multivariate Analysis System. Applied Biostatistics, Port Jefferson, New York

11. Pestana MH, Gageiro JN (2000) Análise de dados para ciências sociais. A complementaridade do SPSS. Edições Sílabo, Lisboa

12. Rodriguez-Otero JL, Paseiro P, Simal J, Cepeda A (1994) Food Chem 49(1):169-171

13. Downey G, Hussey K, Kelly JD, Walshe TF, Martin PG (2005) Food Chem 91(2):347-354

14. Silva LR, Videira R, Monteiro AP, Valentão P, Andrade PB (2009) Microchem J 93(1):73-77

15. González-Miret ML, Terrab A, Hernanz D, Fernández-Recamales MA, Heredia FJ (2005) J Agric Food Chem 53:2574-2580

16. Nozal Nalda MJ, Yague JLB, Calva JCD, Gomez MTM (2005) Anal Bioanal Chem 382(2):311-319

17. Pérez RA, Sánchez-Brunete C, Calvo RM, Tadeo JL (2002) J Agric Food Chem 50(9):2633-2637

18. Baroni MV, Nores ML, Díaz MDP, Chiabrando GA, Fassano JP, Costa C, Wunderlin DA (2006) J Agric Food Chem 54(19):7235-7241

19. Manyi-Loh CE, Ndip RN, Clarke AM (2011) Int J Mol Sci 12(2):9514-9532

20. Wolski T, Tambor K, Rybak-Chmielewska H, Kedzia B (2006) J Apic Sci 50(2):115-126

21. Salgueiro LR, Vila R, Tomi F, Figueiredo AC, Barroso JG, Cañigueral S, Casanova J, da Proença AP, Adzet T (1997) Phytochemistry 45(2):307-311

22. Cavaleiro C, Salgueiro L, Barroso JG, Figueiredo AC, Pedro LG, Fontinha SS, Bighelli A, Casanova J, Looman A, Scheffer JJC (2002) Flavour Fragr J 17(2):111-114

23. Linstrom PJ, Mallard WG (eds) NIST chemistry webbook, NIST standard reference database number 69, National Institute of Standards and Technology, Gaithersburg MD, 20899, http://webbook.nist.gov. Accessed May 2015

24. Joulain D, Köning WA (1998) The atlas of spectral data of sesquiterpene hydrocarbons. E.B.-Verlag, Hamburg 